\title{
Letter to "High biochemical recurrence rate after withdrawal of cabergoline in prolactinomas: is it necessary to restart treatment?"
}

\author{
Burcu Candemir $\mathbb{D}^{1} \cdot$ Şafak Akın ${ }^{1}$
}

Received: 22 October 2020 / Accepted: 24 November 2020 / Published online: 4 January 2021

(c) Springer Science+Business Media, LLC, part of Springer Nature 2021

We read the manuscript entitled "High biochemical recurrence rate after withdrawal of cabergoline in prolactinomas: is it necessary to restart treatment?" with great interest and pleasure [1]. First of all, we would like to congratulate the authors of this article. We thought some points should be clarified so we decided to add some helpful comments on this article.

(1) Currently, the primary treatment in prolactinomas is pharmacological with dopamine agonists according to current guidelines. However, to our knowledge, there is no indication for treatment in patients with macroprolactinemia usually [2]. As stated in the study, macroprolactinemia was detected in 8 of 14 patients in group A (with hyperprolactinemia recurrence, asymptomatic premenopausal women without clear-cut biochemical evidence of hypogonadism and not desiring pregnancy). Were the macroprolactin levels measured in all patients at the time of diagnosis? Shouldn't these patients be followed without treatment from the beginning? Were there any clinical findings that required treatment in these patients before enrollment in the study?

(2) We were confused by some of the information about comparing patients in remission with patients with recurrence. Fourteen of 16 patients who were in remission had tumor shrinkage $>50 \%$. How were these rates in the other two patients? In addition, tumor shrinkage was $>50 \%$ in 28 of 34 patients with recurrence. How was the change in tumor size of the other 6 patients? Why was the treatment discontinued although there was no tumor shrinkage of $>50 \%$ in

Burcu Candemir

bilalogluburcu@gmail.com

1 University of Health Sciences, Gulhane Faculty of Medicine, Department of Endocrinology and Metabolism, Ankara, Turkey these patients? Whereas, the cessation criteria of the treatment in the method section were as follows; continuous treatment with cabergoline for at least 2 years, normalization of PRL concentrations, and significant reduction in tumor size $(\geq 50 \%)$.

In conclusion, this study allowed us to reconsider the criteria for restart of pharmacological treatment in patients with recurrence of hyperprolactinemia after treatment cessation. However, describing specific indications of discontinuation medical treatment and follow-up strategy after cessation may prevent clinicians from misunderstanding about this procedure.

\section{Compliance with ethical standards}

Conflict of interest The authors declare that they have no conflict of interest.

Publisher's note Springer Nature remains neutral with regard to jurisdictional claims in published maps and institutional affiliations.

\section{References}

1. E. Espinosa-Cárdenas, M. Sánchez-García, C. Ramírez-Rentería, V. Mendoza-Zubieta, E. Sosa-Eroza, M. Mercado, High biochemical recurrence rate after withdrawal of cabergoline in prolactinomas: is it necessary to restart treatment? Endocrine 70(1), 143-149 (2020). https://doi.org/10.1007/s12020-020-02388-0

2. S. Melmed, F.F. Casanueva, A.R. Hoffman, D.L. Kleinberg, V.M. Montori, J.A. Schlechte, J.A. Wass, Diagnosis and treatment of hyperprolactinemia: an Endocrine Society clinical practice guideline. J. Clin. Endocrinol. Metab. 96(2), 273-288 (2011). https://doi. org/10.1210/jc.2010-1692 